\title{
Quantum Theory and Its Effects on Novel Corona-Virus
}

\author{
Shahzad Aasim \\ Haldia Institute of Fundamental Research, Kashmir Advanced Scientific Research Centre (KASRC), Kolkata, India \\ Email: drshahzadaasim4@gamil.com, chairmankasrc@gmail.com
}

How to cite this paper: Aasim, S. (2020) Quantum Theory and Its Effects on Novel Corona-Virus. Journal of Quantum Information Science, 10, 36-42.

https://doi.org/10.4236/jqis.2020.102004

Received: May 2, 2020

Accepted: June 27, 2020

Published: June 30, 2020

Copyright (C) 2020 by author(s) and Scientific Research Publishing Inc. This work is licensed under the Creative Commons Attribution International License (CC BY 4.0).

http://creativecommons.org/licenses/by/4.0/

\begin{abstract}
Emerging infectious viral diseases are a major threat to humankind on earth, containing emerging and re-emerging pathogenic physiognomies has raised great public health concern. This study aimed at investigating the global prevalence, biological and clinical characteristics of novel Corona-virus, Wuhan China (2019-nCoV), Severe Acute Respiratory Syndrome Corona-virus (SARS$\mathrm{CoV}$ ), and Middle East Respiratory Syndrome Corona-virus (MERS-CoV) infection outbreaks [1]. Currently, novel Corona-virus disease COVID-2019 is already pandemic and causing havoc throughout the world. Scientific community is still struggling to come out with concrete therapeutic measures against this disease and development of its vaccine is far off from sight in the immediate near future. However, humanity will be put to such pressures very often in the near future and given the present circumstances, what we can expect from the scientific world now? I think QIT (Quantum Information Theory) has an answer to this question. One of the very basic mechanisms that every infectious virus follows to infect is the entry of the virus through cell surface receptors, engulfing, un-coating of viral genome and its transcription to form multiple copies and translation to form viral proteins and coating of viral genome to form multiple copies of the viral particles and then of course the cell bursting to infect other cells. This very basic mechanism does not occur randomly but through a regulated and more dynamic process which we may call coding and decoding of information through reduction in error or noise.
\end{abstract}

\section{Keywords}

COVID-19, Corona-Virus, QIT, SARS-CoV, Iobits, Neobits

\section{Introduction}

Lots of discoveries have taken place in scientific world and among them, the vi- 
rus is one of the significant ones however it appears the smallest. A virus, according to Wikipedia Encyclopedia, is a submicroscopic infectious agent that replicates only inside the living cells of an organism. Viruses can infect all types of life forms, from animals and plants to microorganisms, including bacteria and archaea. Now the question is even in the least possible form, its work is beyond the imagination. It is hard to understand the nature of virus, its configuration and the cause of manifestation is an ordeal and even dreadful. Although, bacteria are the tiniest living organisms found yet but this virus is even tinier and also found in bacteria's [2]. Now the question is even in the tiniest form, how does it exist and what is the mode of manifestation of this virus? Even though there are different types of viruses in the world, this particular one has to be watched very keenly and to study its structure we need to go through microscopic study for which we use electron microscope or electron X-ray techniques and hence, we learn that this living organism works exactly like the others i.e. its structure is like a bacteria composing protein and lipid membranes and DNA/RNA which are also found in different shapes and sizes. Now our point is what makes this organism the tiniest [3]?

Scientifically, it has been proven that it contains RNA which works like a code and acts as a source in its formation. In other words, it basically forms from the RNA. But the point is how the RNA actually initiates when later acquires mass and takes the shape of virus [4]. After that, this virus works in two ways initially, in recessive mode and next, in dominant mode i.e. this virus keeps on forming an unmatched track and at the same time does its work. The other virus which is primarily in the recessive mode but also looks for the host and then works as the dominant factor i.e. it looks for the target to complete its task [5]. But the question is that, how does RNA which is the basic source of its creation, build? To understand this, one has to take aid of quantum information theory that helps in understanding the internal information of the RNA, its configuration and its mode of manifestation. The internal information of the RNA is extremely different from the laws which are identified and acknowledged, it has its own laws and mechanisms that play a key role in acquiring mass wherein super energy mechanism/super time mechanism and super dimensional mechanism are amazing. After interpreting these three laws, let's know how super energy that is in the non-baryonic form with the help of super time and super dimensional mechanism converts into baryonic form? Until, these three laws aren't cognized, formation of this virus won't be grasped.

\section{Quantum Information Theory}

It is such information which plays an important role in formation of matter that actually is an energy and later with other formulas like super energy, super time and super dimension concepts, changes from non-existing form/non-baryonic form to existing form [6]. This information is actually in hidden geometrical form which we need to understand e.g. it is necessary that every matter contains 
some information it is because of this information, the matter exists. We have also framed a mathematical equation for this which actually works same as the mechanism found inside the matter at micro level and it has the same functions like the hereditary information has, at the micro level. Since, it is in non-existing form but on quantum level, we can form a quantum information equation that helps in the formation of hereditary information. As we know, hereditary information contains codes which internally are of different charges or have a dual nature. They work as small bits which are linked to each other in order to make a larger bit which has been named as cubit or we can also claim that information is formed on the basis of the smaller bits. These bits of different nature combine to form a sort of pattern i.e. the equation that we call as Nano-bit and Iobit means iobit-1 and iobit-2 forms Nano-bit and these Nano-bits later form the precursor of positive and negative charge to make unique cubit. Similarly the other iobits also form different types of Nano-bits and later these Nano-bits combine to form a cubit which acquires a unique charge and this charge later can be differentiated into plus and minus. Now the question is what this plus and minus actually is? This is also a different mechanism which we can understand from super energy concept, super time and super dimension concept and it works like lock and key methodology and it later interacts with the bit of a particular configuration and this method continues till the matter is formed to which we assign different names e.g. plus means positive charge and minus means negative charge, sometime it may also have dual nature. This can be understood by a configuration or geometrical structural information.

\section{QIT and Its Role}

Actually, the quantum information which is present in it also carries the shape of its manifestation or we can say that quantum information becomes the source of its designing which later takes a geometrical shape [7]. Even though it is present in geometrical information, but it is in non-baryonic/non-existing form initially and later converts into baryonic form. It means that if we will talk about the world of particles, these particles play an important role in the formation of a structure but along with its structure it also has a particular design/pattern which is present in these quantum information bits and play vital role in the designing of the structure or we can say that in any living or non-living matter there is a particular code for its structure [8]. The plant right from its first particle i.e. nucleic code till it gets developed into a complete plant, is controlled by genetic code and the hereditary of this plant works through the quantum information which means that the structure/manifestation of all the living organisms/matter is present in their codes and its configuration is hidden in its information.

\section{Statistical Analysis}

Whenever, the error or noise becomes more absurd, recombination oramplifica- 
tion of signal occurs which results in a more appropriate information rendered to be coded and decoded in the next phase of infection. This has happened in this virus as follows: SARS-CoV1 TO SARS-CoV2.

The Quantum Information theory is superimposed in two interdependent states we may call it non-infective say 0 and infective state say 1 . We may have ample number of states between non-infective and infective states. The virus is both infective and non-infective at the same time $t=0$, however it may exist in either of these forms at $t>0$. So temporal aspect of the virus cycle is important to note. A corollary can be drawn as at $t=0.01$, at $t>0,0 \ldots . .1$.

Let's have a look at this equation,

$$
\begin{gathered}
I=\text { iobits } \\
N=\text { Neobits } \\
C=\text { Cubits } \\
T=\text { Charge (+ve, }-\mathrm{ve}) \\
T=C=N=I \\
T=C=\left(N_{1}+N_{2}\right)=\left(I_{1}+I_{2}\right) \\
T=C 4\left(I_{1}+I_{2}\right)+2\left(N_{1}+N_{2}\right) \\
T=C=\left(N_{1}=I_{1}+I_{2}+I_{3}+I_{4}\right)+\left(N_{2}+I_{5}+I_{6}+I_{7}+I_{8}\right) \\
T=C=N_{1}\left(I_{1-4}\right)+N_{2}\left(I_{5-8}\right) \\
T=C \\
C=N_{1}+N_{2} \\
N_{1}=\left(I_{1}+I_{2}\right) \\
N_{2}=\left(I_{3}+I_{4}\right) \\
C=N_{1}\left(I_{1}+I_{2}\right)+N_{2}\left(I_{3}+I_{4}\right) \\
T=C=N_{1}\left(I_{1}+I_{2}\right)+N_{2}\left(I_{3}+I_{4}\right) \\
T=C=N_{1}\left(I_{1}+I_{2}\right)+N_{2}\left(I_{3}+I_{4}\right) \\
T=C_{1}+C_{2}=N_{1}\left(I_{1}+I_{2}\right)+N_{2}\left(I_{2}+I_{4}\right)
\end{gathered}
$$

So, this superimposed information can be decoded in advance before any virus becomes infectious and communicable. Here is the diagram that makes it clearer in Figure 1.

\section{Conclusion}

That quantum phenomena might be observable in the messy world of living systems is historically a pejorative idea. While quantum theories accurately describe the behavior of the individual particles making up all matter, scientists have long presumed that the mass action of billions of particles jostling around at ambient temperature drowns out any weird quantum effects and is better explained by the more familiar rules of classical mechanics formulated by Isaac Newton and 


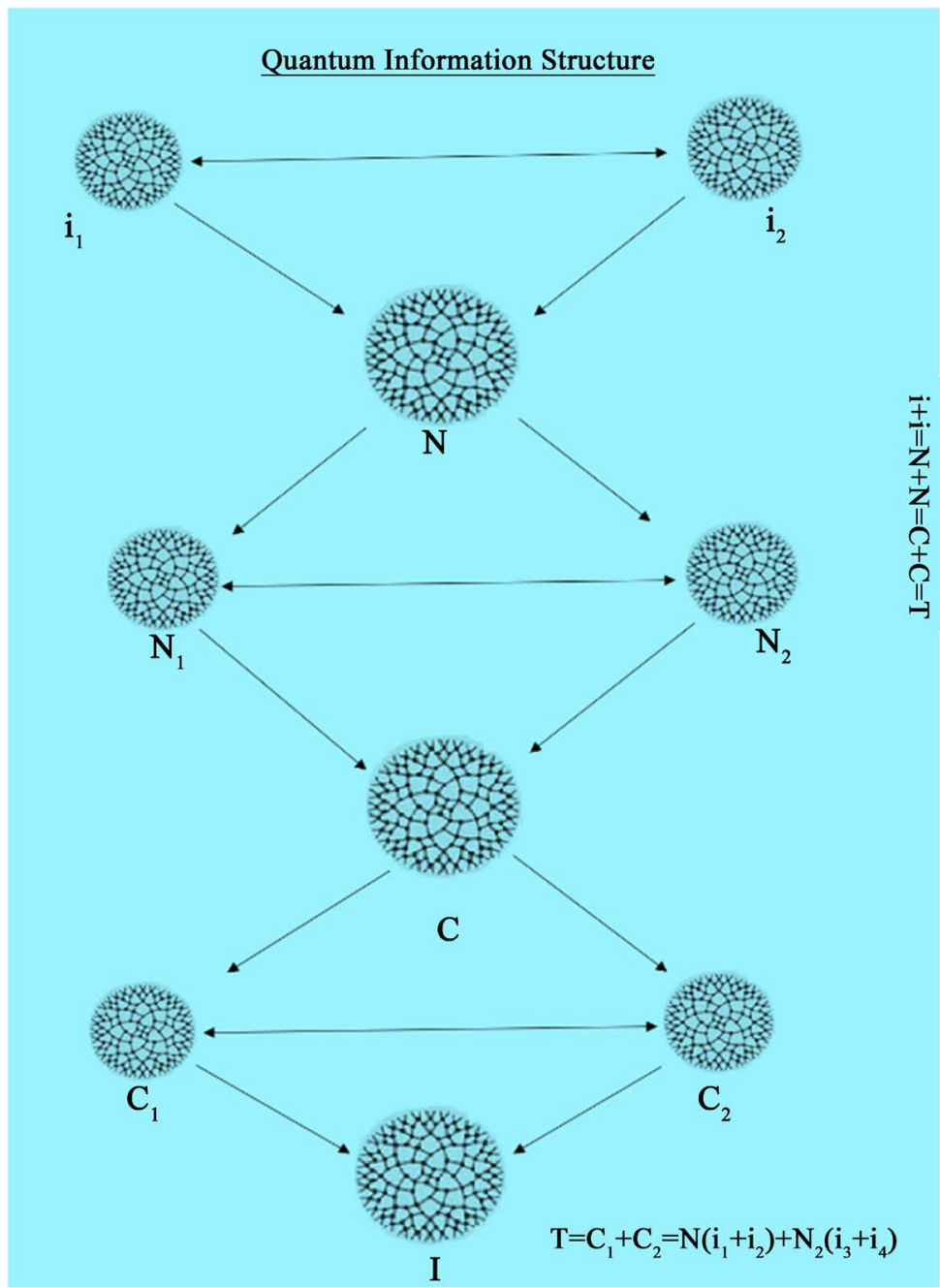

Figure 1. QIT Structure layout.

others [9].

1) Indeed, researchers studying quantum phenomena often isolate particles at temperatures approaching absolute zero-at which almost all particle motion grinds to a halt-just to quash the background noise [10].

2) "The warmer the environment is, the busier and noisy it is, the quicker these quantum effects disappear", says University of Surrey theoretical physicist Jim Al-Khalili, who coauthored a 2014 book called Life on the Edge that brought so-called quantum biology to a lay audience. "So it's almost ridiculous, counterintuitive, that they should persist inside cells. And yet, if they do-and there's a lot of evidence suggesting that in certain phenomena they do-then life must be doing something special [11]".

3) Al-Khalili and Vedral are part of an expanding group of scientists now arguing that effects of the quantum world may be central to explaining some of biology's greatest puzzles-from the efficiency of enzyme catalysis to avian navigation to human consciousness-and could even be subject to natural selection [12]. 
4) In the words of Chiara Marletto "The whole field is trying to prove a point", a University of Oxford physicist who collaborated with Coles and Vedral on the bacteria-entanglement paper. "That is to say, not only does quantum theory apply to these [biological systems], but it's possible to test whether these [systems] are harnessing QIT to perform their functions [13]".

5) Outbreaks of emerging and reemerging pathogens across the globe can be prevented with the help of QIT to minimize the disease burden locally and globally [14].

\section{About the Author}

Dr. Shahzad Aasim is a renowned Scientist working as Director at Haldia Institute of Fundamental Research, Kolkatta. He is also the Chairman of Kashmir Advanced Scientific Research Centre (KASRC). His research includes how Quantum theory reveals the Secrets of Nature.

\section{Conflicts of Interest}

The author declares no conflicts of interest regarding the publication of this paper.

\section{References}

[1] Zhong, N.S., Zheng, B.J., Li, Y.M., Poon, L.L.M., Xie, Z.H., Chan, K.H., Li, P.H., Tan, S.Y., Chang, Q., Xie, J.P., Liu, X.Q., Xu, J., Li, D.X., Yuen, K.Y., Peiris, J.S.M. and Guan, Y. (2003) Epidemiology and Cause of Severe Acute Respiratory Syndrome (SARS) in Guangdong, China, in February 2003. The Lancet, 362, 1353-1358. https://doi.org/10.1016/S0140-6736(03)14630-2

[2] Cha, Y., et al. (1989) Hydrogen Tunneling in Enzyme Reactions. Science, 243, 1325-1330. https://doi.org/10.1126/science.2646716

[3] Doll, K.M., et al. (2003) The First Experimental Test of the Hypothesis that Enzymes Have Evolved to Enhance Hydrogen Tunneling. Journal of the American Chemical Society, 125, 10877-10884. https://doi.org/10.1021/ja030120h

[4] Hu, S., et al. (2019) Biophysical Characterization of a Disabled Double Mutant of Soybean Lipoxygenase: The "Undoing" of Precise Substrate Positioning Relative to Metal Cofactor and an Identified Dynamical Network. Journal of the American Chemical Society, 141, 1555-1567. https://doi.org/10.1021/jacs.8b10992

[5] Hong, N.-S., et al. (2018) The Evolution of Multiple Active Site Configurations in a Designed Enzyme. Nature Communications, 9, Article No. 3900. https://doi.org/10.1038/s41467-018-06305-y

[6] Engel, G.S., et al. (2007) Evidence for Wavelike Energy Transfer through Quantum Coherence in Photosynthetic Systems. Nature, 446, 782-786.

https://doi.org/10.1038/nature05678

[7] Panitchayangkoon, G., et al. (2010) Long-Lived Quantum Coherence in Photosynthetic Complexes at Physiological Temperature. PNAS, 107, 12766-12770. https://doi.org/10.1073/pnas.1005484107

[8] Collini, E., et al. (2010) Coherently Wired Light-Harvesting in Photosynthetic Marine Algae at Ambient Temperature. Nature, 463, 644-647. https://doi.org/10.1038/nature08811 
[9] Calhoun, T.R., et al. (2009) Quantum Coherence Enabled Determination of the Energy Landscape in Light-Harvesting Complex II. The Journal of Physical Chemistry $B, 113,16291-16295$. https://doi.org/10.1021/jp908300c

[10] Duan, H.-G., et al. (2017) Nature Does Not Rely on Long-Lived Electronic Quantum Coherence for Photosynthetic Energy Transfer. PNAS, 114, 8493-8498. https://doi.org/10.1073/pnas.1702261114

[11] Thyrhaug, E., et al. (2018) Identification and Characterization of Diverse Coherences in the Fenna-Matthews-Olson Complex. Nature Chemistry, 10, 780-786. https://doi.org/10.1038/s41557-018-0060-5

[12] Harrop, S.J., et al. (2014) Single-Residue Insertion Switches the Quaternary Structure and Exciton States of Cryptophyte Light-Harvesting Proteins. PNAS, 111, E2666-E2675. https://doi.org/10.1073/pnas.1402538111

[13] Ritz, T., et al. (2000) A Model for Photoreceptor-Based Magnetoreception in Birds. Biophysical Journal, 78, 707-718. https://doi.org/10.1016/S0006-3495(00)76629-X

[14] Ritz, T., et al. (2004) Resonance Effects Indicate a Radical-Pair Mechanism for Avian Magnetic Compass. Nature, 429, 177-180.

https://doi.org/10.1038/nature02534 